\title{
User-interactive electronic skin for instantaneous pressure visualization
}

\author{
Chuan Wang ${ }^{1,2,3 \dagger}$, David Hwang ${ }^{1,2,3}$, Zhibin $\mathrm{Yu}^{1,2,3}$, Kuniharu Takei ${ }^{1,2,3}$, Junwoo Park ${ }^{4}$, Teresa Chen ${ }^{4}$, \\ Biwu $\mathrm{Ma}^{3,4}$ and Ali Javey ${ }^{1,2,3 \star}$
}

\begin{abstract}
Electronic skin (e-skin) presents a network of mechanically flexible sensors that can conformally wrap irregular surfaces and spatially map and quantify various stimuli ${ }^{1-12}$. Previous works on e-skin have focused on the optimization of pressure sensors interfaced with an electronic readout, whereas user interfaces based on a human-readable output were not explored. Here, we report the first user-interactive e-skin that not only spatially maps the applied pressure but also provides an instantaneous visual response through a built-in active-matrix organic light-emitting diode display with red, green and blue pixels. In this system, organic light-emitting diodes (OLEDs) are turned on locally where the surface is touched, and the intensity of the emitted light quantifies the magnitude of the applied pressure. This work represents a system-on-plastic ${ }^{4,13-17}$ demonstration where three distinct electronic componentsthin-film transistor, pressure sensor and OLED arrays-are monolithically integrated over large areas on a single plastic substrate. The reported e-skin may find a wide range of applications in interactive input/control devices, smart wallpapers, robotics and medical/health monitoring devices.
\end{abstract}

Although both passive $e^{6,8,12}$ and active-matrix ${ }^{1,2,5,9}$ designs can be used for enabling the predicted user-interactive e-skins, the active-matrix design is advantageous as it minimizes signal crosstalk and thereby offers better spatial resolution and contrast, and a faster response. In the active-matrix backplane circuitry, each pixel is controlled by a thin-film transistor (TFT) that acts as a switch for addressing either current- or voltage-driven devices. Here, we incorporate the active-matrix design into the e-skin by using semiconductor-enriched nanotubes ${ }^{18}$ as the channel material of the TFTs. Carbon nanotube networks are proven to be a promising material platform for high-performance TFTs (refs 9,17,19-21) with high current drives needed for switching OLEDs (ref. 22). A schematic structure of a pixel of the user-interactive e-skin with an integrated TFT, OLED and pressure sensor is depicted in Fig. 1a. Each pixel in the active-matrix consists of a nanotube TFT with the drain connected to the anode of an OLED. The OLED uses a simple bilayer structure ${ }^{23}$ and the colour of the emitted light is controlled by using different emissive layer materials (details in the Methods). In this work, red, green, blue and yellow colours are demonstrated. On top of the OLEDs, a pressure-sensitive rubber $^{1,5,24,25}$ (PSR) is laminated, which is in electrical contact with the cathode (that is, top contact) of the OLED at each pixel. The top surface of the PSR is coated with a conductive silver ink to act as the ground contact. Here, the conductivity of the PSR increases by an applied pressure $e^{1,5,24,25}$ that subsequently results in the underlying OLED turning on. As illustrated in Fig. 1b, the single-pixel circuitry is integrated into an active-matrix array. The resulting system-on-plastic provides a touch user interface, allowing the pressure profile to be both spatially mapped and visually seen. Optical micrographs of one pixel and the entire array $(16 \times 16$ pixels with a size of $\sim 3 \times 3.5 \mathrm{~cm}^{2}$ ) of the fabricated device are shown in Fig. 1c,d, respectively, with the latter depicting the mechanical flexibility of the system.

Given the large number of active components needed for the user-interactive e-skin, it is essential to use a process scheme that leads to a high yield of uniform devices on a plastic substrate. The active-matrix back-plane was fabricated on a polyimide-coated Si wafer followed by OLED processing. First, a thin $(\sim 24 \mu \mathrm{m})$ polyimide layer is spin-coated and cured on a Si handling wafer. Next, the wafer is processed by conventional microfabrication steps, such as photolithography and metallization, to pattern and deposit the various layers. After the completion of fabrication, the polyimide layer with the devices on top was delaminated from the handling wafer ${ }^{5,9,17}$, resulting in a highly bendable systemon-plastic. The step-by-step details of the fabrication process are presented in the Methods and Supplementary Fig. S1. This fabrication approach results in highly uniform devices by using the well-established microfabrication process technology. Although the sample size is limited to that of the handing wafer, the entire fabrication process is compatible with liquid-crystal-display fabrication processes, which could allow metre-scale processing of the proposed system in the future.

We first characterize the electrical performance of the flexible carbon nanotube TFT active-matrix backplane. The transfer characteristics $\left(I_{\mathrm{DS}}-V_{\mathrm{GS}}\right)$ of 20 representative TFTs $(L=20 \mu \mathrm{m}$, $W=2,000 \mu \mathrm{m})$ measured at $V_{\mathrm{DS}}=-5 \mathrm{~V}$ are presented in Fig. 2a. The transistors exhibit a uniform performance in terms of on-current $\left(I_{\mathrm{on}}\right)$, transconductance $\left(g_{\mathrm{m}}\right)$ and mobility (Supplementary Fig. S2), which is attributed to the uniform nanotube networks obtained using the assembly method applied in this work ${ }^{9,17}$. The mean and standard deviation values for $I_{\mathrm{on}}, g_{\mathrm{m}}$ and field-effect mobility are $3.6 \pm 0.3 \mathrm{~mA}, 1.0 \pm 0.1 \mathrm{mS}$ and $20 \pm 2 \mathrm{~cm}^{2} \mathrm{~V}^{-1} \mathrm{~s}^{-1}$, respectively. On the other hand, the off-state current (and thereby the $I_{\text {on }} / I_{\text {off }}$ ) of the transistors exhibits a larger device-to-device variation as depicted in Fig. 2a, ranging from $\sim 0.1$ to $3 \mu \mathrm{A}$, with an average value of $0.84 \pm 0.80 \mu \mathrm{A}$. Although this variation is acceptable for the system demonstration of this work, it may be further improved in the future by minimizing contaminants in the devices by using higher-purity nanotube samples, and performing the entire device processing in a cleanroom environment.

\footnotetext{
${ }^{1}$ Department of Electrical Engineering and Computer Sciences, University of California, Berkeley, California 94720, USA, ${ }^{2}$ Berkeley Sensor and Actuator Center, University of California, Berkeley, California 94720, USA, ${ }^{3}$ Materials Sciences Division, Lawrence Berkeley National Laboratory, Berkeley, California 94720, USA, ${ }^{4}$ The Molecular Foundry, Lawrence Berkeley National Laboratory, Berkeley, California 94720, USA. 'Present address: Department of Electrical and Computer Engineering, Michigan State University, East Lansing, Michigan 48824, USA. *e-mail: ajavey@eecs.berkeley.edu
} 


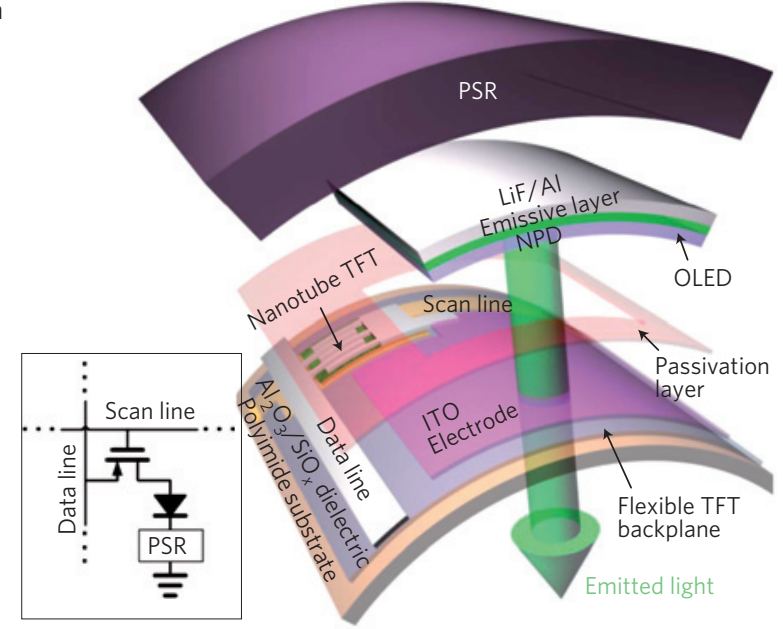

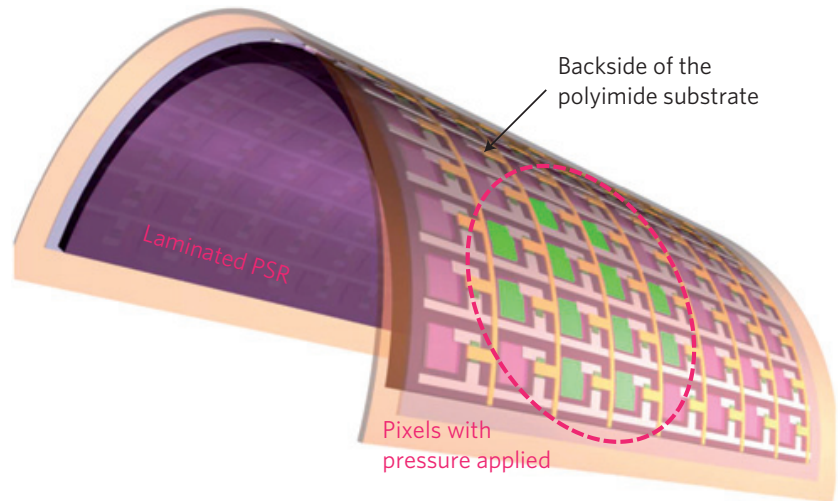

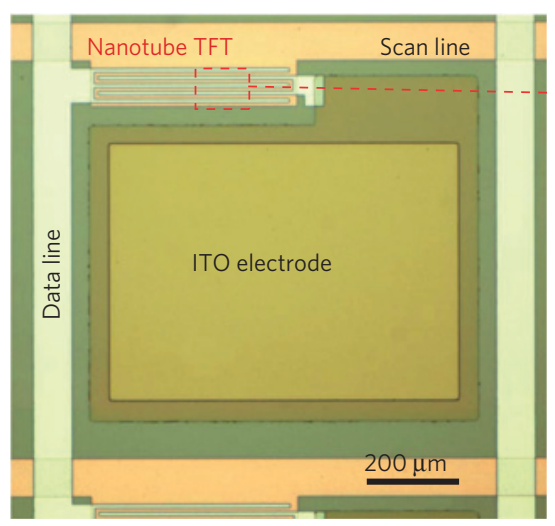

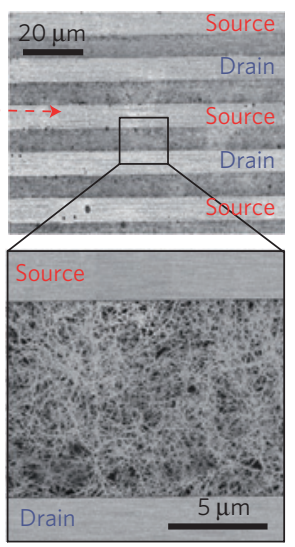

d

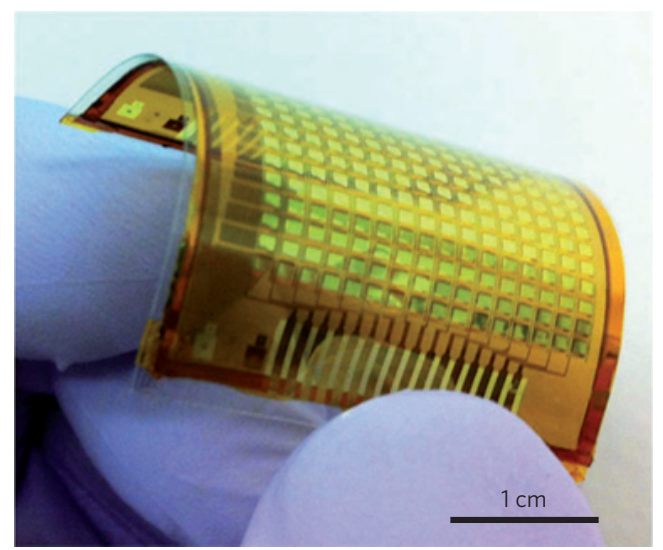

Figure 1 | Concept and structure of the user-interactive e-skin. a, Schematic layout of a single pixel, consisting of a nanotube TFT, an OLED and a pressure sensor (PSR) integrated vertically on a polyimide substrate. b, Schematic diagram of an array of pixels $(16 \times 16)$ functioning as an interactive e-skin, capable of spatially mapping and visually displaying an applied pressure profile. c, Optical micrograph of a fabricated pixel before the integration of the OLED and PSR. The drain of the TFT is connected to an ITO pad, which serves as the anode electrode for the corresponding OLED. Scanning electron micrographs of the active area of a nanotube TFT are also shown. d, Optical photograph of a fully fabricated interactive e-skin containing $16 \times 16$ pixels with a size of $\sim 3 \times 3.5 \mathrm{~cm}^{2}$.

Furthermore, the off-state current values may be further reduced by using higher enriched semiconducting carbon nanotube samples (99\% semiconductor-enriched used in this work). Notably, the high current-drive of nanotube TFTs is essential for driving OLED pixels (see Supplementary Information for more detailed analysis) and the uniformity is critical for achieving high-yield macroscale electronic systems. In addition, the active-matrix backplane also offers excellent flexibility without degradation to the device performance as shown in the Supplementary Fig. S3) and our previous studies ${ }^{9,17}$.

The characteristics of the standalone OLEDs are also characterized. The electroluminescence spectra of the OLEDs with four different colours (red, yellow, green, blue) are measured using a spectrofluorometer and the results are plotted in Fig. 2b. By simply changing the emissive layer material, the emitted peak wavelength can be adjusted to $489,523,562$ or $601 \mathrm{~nm}$. The exact device structure and materials, and the corresponding photographs of the OLEDs with different colours, are presented in Supplementary Fig. S4. The OLEDs' current and brightness as a function of the applied voltage are also measured and plotted in Fig. $2 \mathrm{c}, \mathrm{d}$, respectively. All 4 types of OLED exhibit clear rectifying $I-V$ characteristics with noticeable light output at an applied bias of $>3 \mathrm{~V}$ (current $\sim 10-20 \mu \mathrm{A}$, area $=4 \mathrm{~mm}^{2}$ ). By interconnecting the anode of an OLED to the drain of a carbon nanotube TFT, a single-pixel OLED control circuit is constructed. Figure 2e,f shows the transfer and output characteristics of a single-pixel circuitry, respectively, with the circuit schematics shown in the insets. By sweeping the gate voltage $\left(V_{\mathrm{G}}\right)$ or the power supply $\left(V_{\mathrm{DD}}\right)$ of the control TFT, the current flowing through the OLED $\left(I_{\mathrm{DD}}\right)$ can be effectively controlled, which translates into the modulation of the OLED brightness, allowing the OLEDs to be turned on and off. The transfer and output characteristics of a single-pixel circuit resemble the ones for the nanotube transistors except that the threshold voltage of the diode $\left(V_{\text {th_OLED }}\right)$ is now introduced into the output characteristics (Fig. 2f) and thereby a $V_{\mathrm{DD}}$ above $3 \mathrm{~V}$ is needed to turn on the circuit.

Arranging the above-described single-pixel OLED control circuitry into a matrix results in a monolithically integrated activematrix OLED (AMOLED) display (Fig. 3a), which is subsequently integrated with pressure sensors for the interactive e-skin. A photograph of a flexible AMOLED display is shown in Fig. 3b. Note that the light is emitted through the semi-transparent polyimide substrate, as the top surface of the sample is covered by the aluminium cathodes of the OLEDs. Figure 3c shows a single-colour (green) flexible AMOLED display with $-5 \mathrm{~V}$ and $10 \mathrm{~V}$ applied to all of the scan and data lines, respectively. Such a configuration allows all of the pixels $(16 \times 16)$ to be turned on, and the measured pixel yield is more than $97 \%$. Figure $3 \mathrm{c}$ also confirms that the evaporated organic and metallic thin films in the display are durable on bending and the 


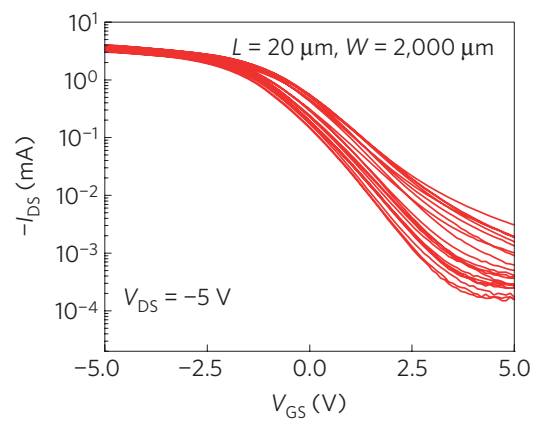

d

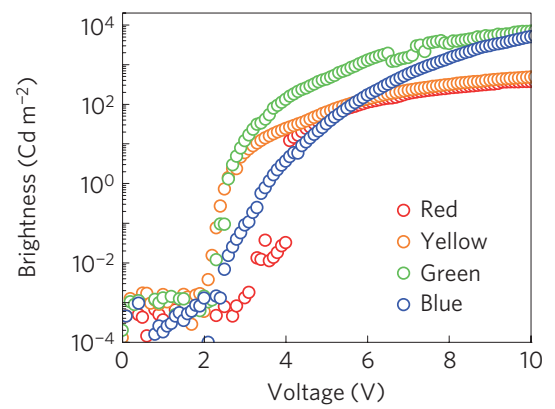

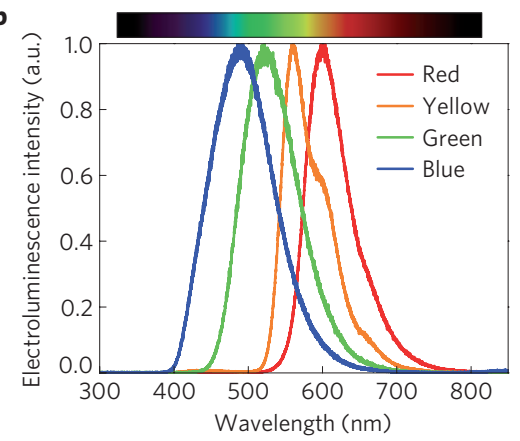

e

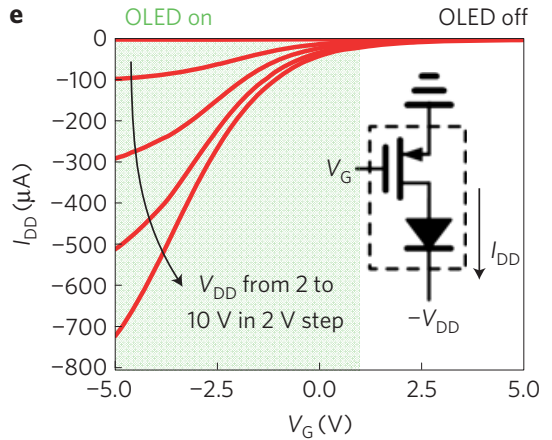

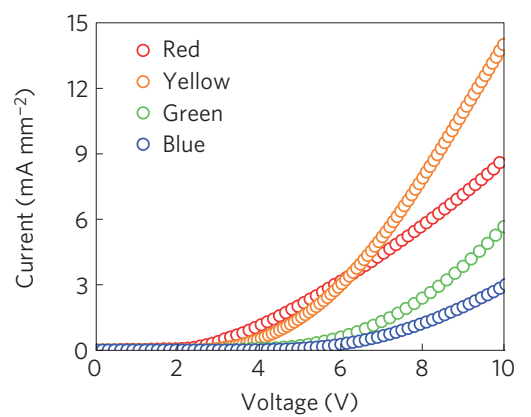

f

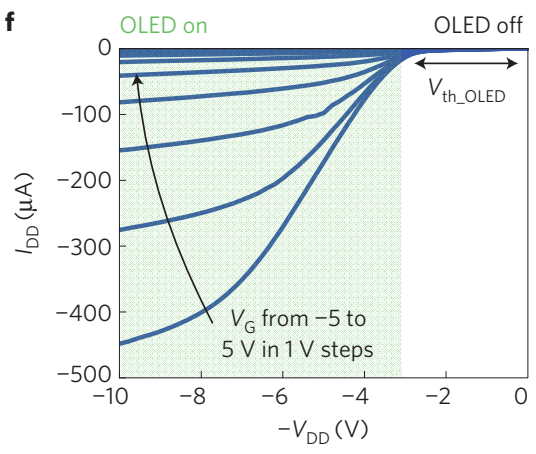

Figure 2 | Electrical characterization of carbon nanotube TFTs and OLEDs. a, Transfer characteristics of 20 different nanotube TFTs in the active-matrix backplane ( $L=20 \mu \mathrm{m}, W=2 \mathrm{~mm}$ ), showing uniform device properties. $\mathbf{b}$, Electroluminescence spectra of red, yellow, green and blue OLEDs (measured at $\sim 7 \mathrm{~V}$ ) obtained by using (pq) 2 Ir (acac), rubrene, $\mathrm{Alq} 3$ and $\mathrm{B}(\mathrm{Alq})$ as the emissive layer, respectively. c, $I-V$ characteristics of the fabricated OLEDs with different colours. d, Brightness as a function of applied voltage for different colour OLEDs. e,f, Transfer (e) and output characteristics (f) of a green OLED

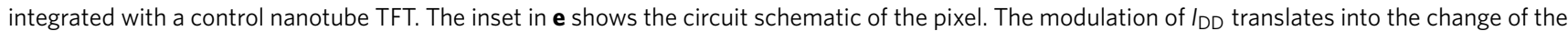
OLED brightness so that the pixel can be turned on and off.

display remains able to function properly under various bending conditions. Furthermore, electrical measurements of an individual pixel under various bending radii are shown in Supplementary Fig. S3a, depicting minimal change up to a radius of $\sim 4 \mathrm{~mm}$. A full-colour flexible AMOLED display (that is red, green, blue OLED pixels monolithically integrated on the same substrate) is also demonstrated through a multi-step evaporation of the various emissive layers. Figure 3d,e shows the full-colour display being fully turned on in the relaxed and bent states, respectively, with a pixel yield of $\sim 85 \%$. Each of the pixels in the display can be individually addressed using the nanotube TFTs as depicted in Fig. 3f. Although single-colour AMOLED displays have been demonstrated previously on rigid glass substrates using carbon nanotube transistors ${ }^{22}$, this paper is the first report of a carbon nanotube-based AMOLED display with red, green and blue colours integrated on a flexible substrate. The work shows the utility of semiconductor-enriched nanotube TFTs for high-performance and bendable displays.

The flexible display is subsequently used in the realization of an interactive e-skin that is capable of spatially mapping and simultaneously responding to the applied pressure. To make the pixels pressure responsive, a PSR is laminated on top of the OLEDs. In this design, the cathode of each OLED is connected to the ground through the PSR. When pressure is applied on the PSR, the tunnelling path between the conductive carbon nanoparticles embedded in the rubber is shortened, resulting in reduced resistance ${ }^{1,5,24,25}$. The resistance change of the PSR modulates the current flowing through the OLED and changes the brightness of the output light. The pressure response of a standalone OLED with a laminated PSR is depicted in Fig. 4 and Supplementary Fig. S5. As pressure is applied, the $I-V$ characteristics of the device undertake pronounced changes (Fig. $4 \mathrm{~b}$ and Supplementary Fig. S5b). Figure 4c and Supplementary Fig. S5c show the current (red trace) and brightness (blue trace) of the OLEDs as a function of the applied pressure. The results indicate that light visible by the naked eye $\left(>1 \mathrm{Cd} \mathrm{m}^{-2}\right)$ is emitted when the pressure is above $\sim 10 \mathrm{kPa}$. Beyond this point, both the current and brightness increase linearly as a function of the applied pressure up to $100 \mathrm{kPa}$. From the slope of the linear response, the sensitivity is extracted to be $\sim 42.7 \mathrm{Cd} \mathrm{m}^{-2} \mathrm{kPa}^{-1}$ for a device area of $\sim 4 \mathrm{~mm}^{2}$. The change in the brightness can be visually seen from the inset photographs of an OLED in Fig. 4c.

Next, the full system is fabricated consisting of $16 \times 16$ pixel arrays of nanotube TFTs, OLEDs and pressure sensors heterogeneously integrated on a polyimide substrate. Cross-sectional and circuit schematics are shown in Fig. 5a,b, respectively. The process involves the fabrication of the nanotube active-matrix backplane, followed by OLEDs, and subsequently the lamination of the PSR on top. The PSR is connected to each pixel through the LiF/Al cathode of the underlying AMOLED. The top surface of the PSR is coated with conductive silver ink as the ground electrode. During the measurements, the scan and data lines are connected to -5 and $10 \mathrm{~V}$, respectively. When no pressure is applied, the resistance of the PSR prevents the current from flowing, thereby all OLEDs are off. The current flow increases and light is emitted locally from the OLEDs by applying pressure. The interactive e-skin can be used to spatially map and visually display the applied pressure profile (Fig. $5 \mathrm{c}-\mathrm{e}$ and Supplementary Movie). Here, transparent polydimethylsiloxane (PDMS) slabs cut into different shapes are used to apply pressure on the sensor array. In Fig. 5e, e-skin devices with green, blue and red colour OLEDs are used to spatially map and display the pressure applied by C-, A- and L-shaped PDMS slabs, respectively. Applying pressure through the letter-shaped PDMS slabs produces recognizable letters with good spatial resolution, limited by the size of the pixels used here. As shown in Fig. 4, the brightness of each OLED represents the magnitude of the local pressure. In addition to the optical visualization, electrical readout can be performed by 


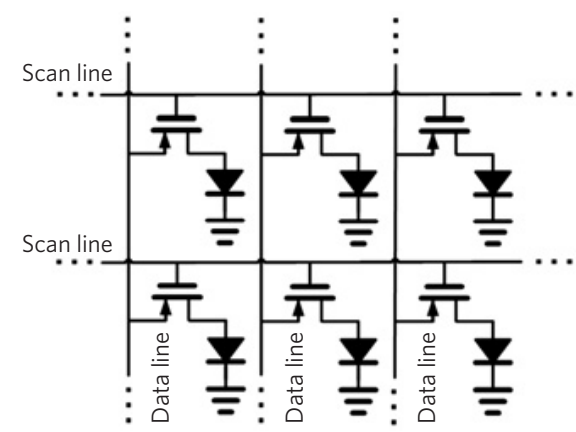

b

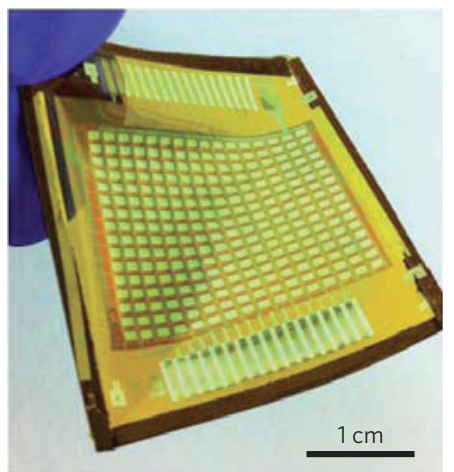

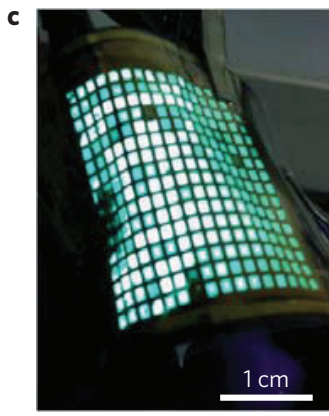
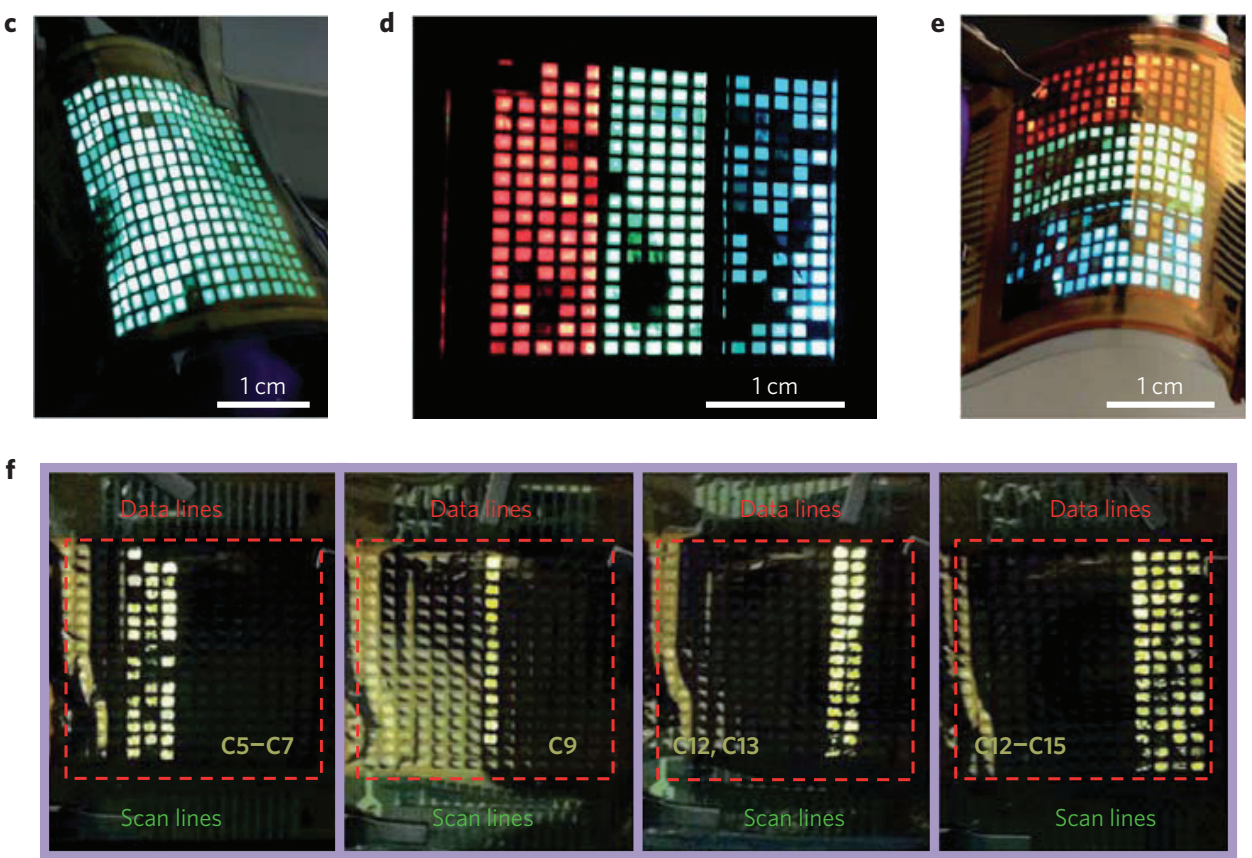

Figure 3 | Flexible full-colour AMOLED display using carbon nanotube TFTs. a, Schematic of the AMOLED display circuitry. $\mathbf{b}$, Photograph of a multi-colour AMOLED display before connecting to the supply voltage. c, Photo of a single-colour (green) AMOLED being fully turned on and bent. Voltages of -5 and $10 \mathrm{~V}$ are applied to all of the scan and data lines, respectively. The pixel yield, as defined by the percentage of OLEDs in the matrix that emit light, is higher than $97 \%$. d, Photograph of a full-colour (red, green, blue) AMOLED display with all pixels being turned on. $\mathbf{e}$, Photo of the same full-colour display shown in $\mathbf{d}$ being bent. f, Photos showing different columns of a single-colour (yellow) AMOLED display being selectively turned on.

a

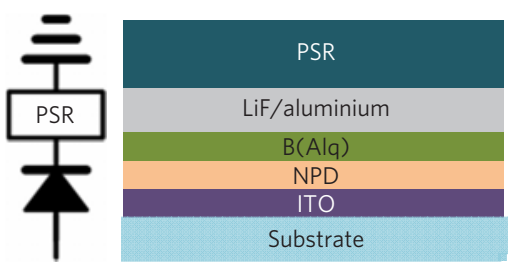

b

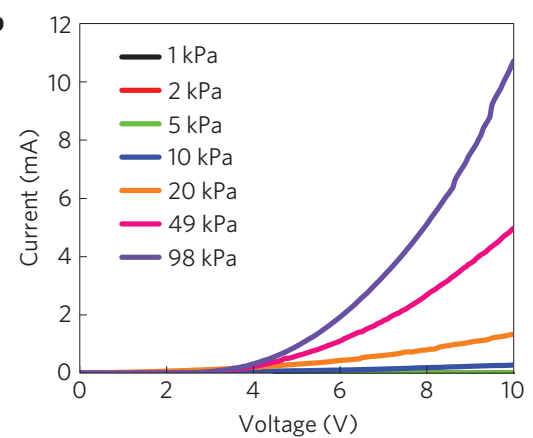

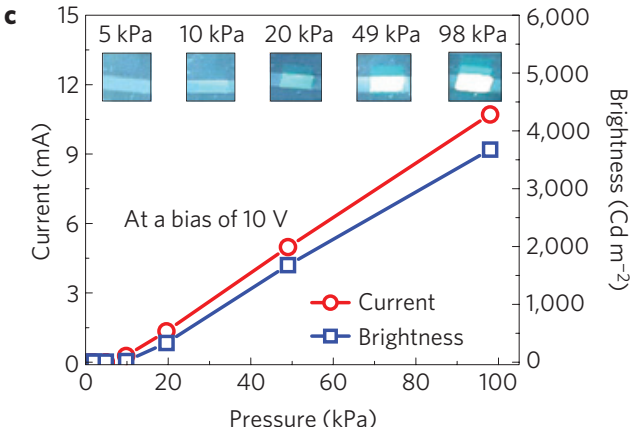

Figure 4 | Pressure response of a standalone OLED with laminated PSR. a, Circuit and cross-sectional schematics of an OLED vertically integrated with a PSR. b, I-V characteristics of the OLED and PSR combination under various applied pressures. c, The current (red trace) and brightness (blue trace) of the OLED as a function of applied pressure at a voltage of $10 \mathrm{~V}$. Inset: photographs of an OLED under various applied pressures.

monitoring the current (that is, resistance) of each pixel as shown in Supplementary Fig. S6. A good correlation between the optical and electrical readout of an applied pressure profile is obtained.

We have characterized the response time of a singlepixel circuit consisting of a nanotube TFT and an OLED
(Supplementary Fig. S7). The measured response time of the circuit is $\sim 1 \mathrm{~ms}$, which is limited by the parasitic capacitance from the metal interconnections and large size OLEDs used in this work. This response time is $\sim 100 \times$ faster than the response time of the PSR $(\sim 0.1 \mathrm{~s})$ as reported in our previous publication ${ }^{5}$. As a result, 

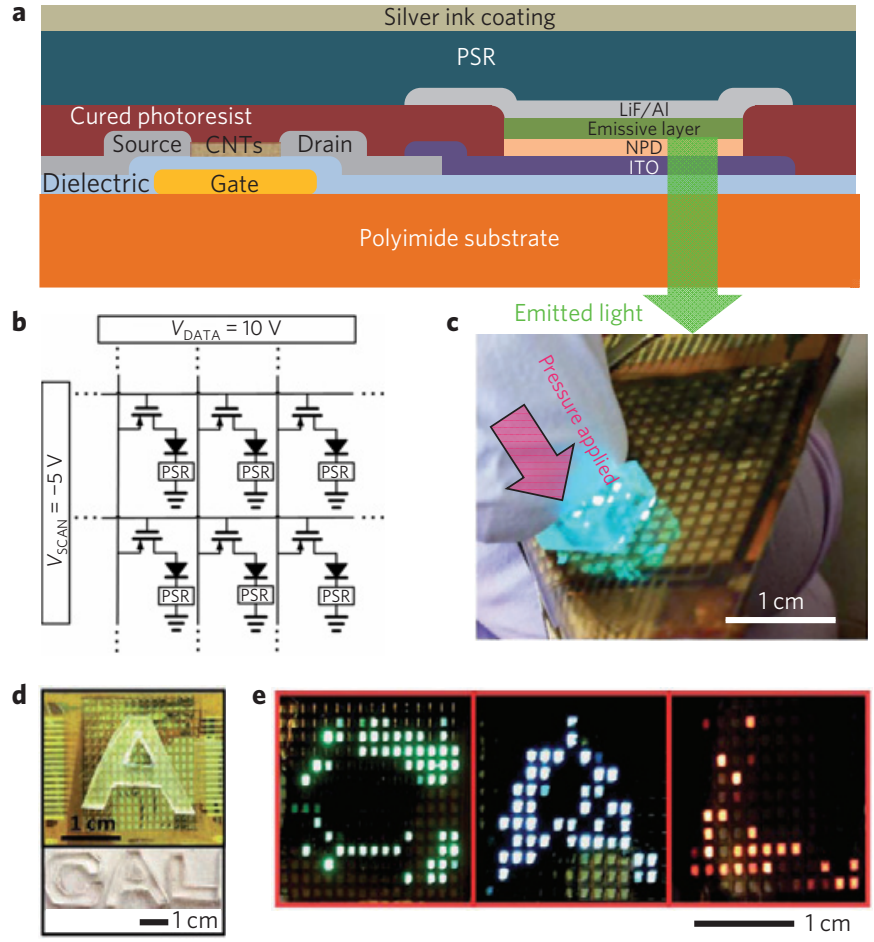

Figure 5 | User-interactive e-skin. a, Cross-sectional schematic showing one pixel of the interactive e-skin device, consisting of a nanotube TFT, an OLED and a pressure sensor. Light from the OLED is emitted through the substrate and the brightness of the OLED is determined by the magnitude of the applied pressure. $\mathbf{b}$, Circuit schematic of the e-skin matrix. c, Photograph of a fabricated device $(16 \times 16$ pixels $)$, showing that light is locally emitted where the surface is touched. Only the pixels being pressed are turned on. $\mathbf{d}$, PDMS slabs with $\mathrm{C}, \mathrm{A}$, and $\mathrm{L}$ shapes are prepared and used to apply pressure onto the sensor array. e, Green, blue and red colour interactive e-skins are used to spatially map and display the pressure applied with C- (left), A- (centre) and L- (right) shaped PDMS slabs, respectively.

for the user-interactive e-skin system, the maximum operating speed is limited by the PSR. This response time is fast enough for most practical sensing and mapping applications. Supplementary Fig. S8 and Movie depict the response time of the full-system under operation by a user.

The work here presents a practical technology platform involving the heterogeneous integration of various electronic, sensor and light-emitting components based on both organic and inorganic materials at a system level on thin plastic substrates. This is the first demonstration of a user-interactive flexible system that can not only detect and spatially map external stimuli (in this case pressure) but also respond with a seamlessly integrated display. This system-on-plastic demonstration enables the sensed pressure profile to be instantaneously visible without the need for sophisticated data acquisition circuits and electronic boards. The pressure sensing used here presents just one example system. In the future, integration of other sensor components ${ }^{26-30}$ can be predicted using a similar platform to enable more sophisticated human-surface interfacing. We predict that the presented platform may find a wide range of applications in automotive control panels, interactive input devices, robotics, and medical and health monitoring devices. Future work along this direction could involve further improving the pixel yield and resolution, incorporation of an encapsulation layer for the OLEDs to enhance their lifetime (Supplementary Fig. S9), and integration of multiple types of sensor element for multifunctional sensing ${ }^{2,7}$. Fully printed TFT backplane, sensor element and polymer OLED arrays could also be explored for covering large areas, such as walls of buildings with interactive wallpapers using the presented e-skin concept.

\section{Methods}

Fabrication of carbon nanotube TFT active-matrix backplane. Polyimide (HD MicroSystems, polyimide-2525) is spin-coated and cured on a silicon handling wafer to serve as the flexible substrate. On top of the polyimide substrate, Ti/Au $(5 / 35 \mathrm{~nm})$ back-gate electrodes (scan lines) are defined by photolithography and lift-off. The gate dielectric consists of $60 \mathrm{~nm}$ of $\mathrm{Al}_{2} \mathrm{O}_{3}$ deposited using atomic layer deposition and $5 \mathrm{~nm}$ of $\mathrm{SiO}_{x}$ deposited using electron-beam evaporation. The wafer is then immersed into poly-L-lysine $(0.1 \%$ wt in water from Sigma Aldrich) for $5 \mathrm{~min}$ to functionalize the $\mathrm{SiO}_{x}$ surface to form an amine-terminated adhesion layer, and the sample is subsequently immersed into the commercially available $0.01 \mathrm{mg} \mathrm{ml}^{-1} 99 \%$ semiconducting nanotube solution (NanoIntegris) for $15 \mathrm{~min}$ followed by deionized water and an isopropanol rinse, and blown dry with nitrogen. After the uniform semiconducting nanotube networks are deposited, the sample is annealed in a vacuum oven at $200^{\circ} \mathrm{C}$ for $1 \mathrm{~h}$ to further clean the surface and remove surfactant residues. Ti/Pd $(0.5 / 40 \mathrm{~nm})$ source-drain contacts (data lines) are then formed by photolithography and a lift-off process, and the carbon nanotube networks are confined to the channel region by patterned etching of all other areas using oxygen plasma. Finally, 50-nm-thick indium tin oxide (ITO) pads connected to the drain of each TFT are defined by photolithography, d.c. sputtering and a lift-off process. The ITO pads are used as the anode electrodes for the subsequently fabricated OLEDs.

Integration with OLEDs and PSR. After the backplane is fabricated, a photoresist layer (thickness $\sim 1 \mu \mathrm{m}$ ) is coated on the surface and rectangular vias with a size of $0.91 \times 0.67 \mathrm{~mm}^{2}$ are patterned on top of the ITO pads. The sample is then heated to $250^{\circ} \mathrm{C}$ in air for $30 \mathrm{~min}$ to anneal the sputtered ITO (to reduce the sheet resistance) and to hard bake the photoresist to serve as an electrical insulation layer. OLEDs with $50 \mathrm{~nm}$ of $4-4^{\prime}$-bis[ $N$-(1-naphthyl)- $N$-phenyl-amino]biphenyl (NPD) as the hole transport layer, $40 \mathrm{~nm}$ of emissive layer, $1 \mathrm{~nm}$ of lithium fluoride (LiF; used to adjust the workfunction) and $100 \mathrm{~nm}$ of aluminium are deposited through a shadow mask by thermal evaporation. OLED fabrication is performed in a nitrogen-filled glovebox with oxygen and moisture levels below $1 \mathrm{ppm}$. Different emissive layer materials are used to obtain OLEDs with different colours: iridium(III)bis(2-phenylquinoline-N,C2')acetylacetonate $\left((\mathrm{pq})_{2} \operatorname{Ir}(\mathrm{acac})\right)$ for red, tris $(8$-hydroxyquinoline $)$ aluminium $\left(\mathrm{Alq}_{3}\right)$ for green, aluminium(III)bis(2-methyl-8-quinolinato)4-phenylphenolate (B(Alq)) for blue, and rubrene for yellow. As a final step, a PSR (PCR Technical) is laminated on the top surface to enable pressure sensing.

Received 7 January 2013; accepted 11 June 2013; published online 21 July 2013

\section{References}

1. Someya, T. et al. A large-area, flexible pressure sensor matrix with organic field-effect transistors for artificial skin applications. Proc. Natl Acad. Sci. USA 101, 9966-9970 (2004).

2. Someya, T. et al. Conformable, flexible, large-area networks of pressure and thermal sensors with organic transistor active matrixes. Proc. Natl Acad. Sci. USA 102, 12321-12325 (2005).

3. Wagner, S. et al. Electronic skin: Architecture and components. Physica E 25, 326-334 (2004).

4. Sekitani, T. \& Someya, T. Stretchable, large-area organic electronics. Adv. Mater. 22, 2228-2246 (2010).

5. Takei, K. et al. Nanowire active-matrix circuitry for low-voltage macroscale artificial skin. Nature Mater. 9, 821-826 (2010).

6. Mannsfeld, S. C. B. et al. Highly sensitive flexible pressure sensors with microstructured rubber dielectric layers. Nature Mater. 9, 859-864 (2010).

7. Kim, D. H. et al. Epidermal electronics. Science 333, 838-843 (2011).

8. Lipomi, D. J. et al. Skin-like pressure and strain sensors based on transparent elastic films of carbon nanotubes. Nature Nanotech. 6, 788-792 (2011).

9. Takahashi, T., Takei, K., Gillies, A. G., Fearing, R. S. \& Javey, A. Carbon nanotube active-matrix backplanes for conformal electronics and sensors. Nano Lett. 11, 5408-5413 (2011).

10. Tee, B. C-K., Wang, C., Allen, R. \& Bao, Z. An electrically and mechanically self-healing composite with pressure- and flexion-sensitive properties for electronic skin applications. Nature Nanotech. 7, 825-831 (2012).

11. Lu, N., Lu, C., Yang, S. \& Rogers, J. Highly sensitive skin-mountable strain gauges based entirely on elastomers. Adv. Funct. Mater. 22, 4044-4050 (2012).

12. Pang, C. et al. A flexible and highly sensitive strain-gauge sensor using reversible interlocking of nanofibres. Nature Mater. 11, 795-801 (2012).

13. Rogers, J. A., Someya, T. \& Huang, Y. Materials and mechanics for stretchable electronics. Science 327, 1603-1607 (2010).

14. Kim, D. H., Xiao, J., Song, J., Huang, Y. \& Rogers, J. A. Stretchable, curvilinear electronics based on inorganic materials. Adv. Mater. 22, 2108-2124 (2010). 
15. Sekitani, T. et al. Organic nonvolatile memory transistors for flexible sensor arrays. Science 326, 1516-1519 (2009).

16. Park, S-I. et al. Printed assemblies of inorganic light-emitting diodes for deformable and semitransparent displays. Science 325, 977-981 (2009).

17. Wang, C., Takei, K., Takahashi, T. \& Javey, A. Carbon nanotube electronics-moving forward. Chem. Soc. Rev. 42, 2592-2609 (2013).

18. Arnold, M. S., Green, A. A., Hulvat, J. F., Stupp, S. I. \& Hersam, M. C. Sorting carbon nanotubes by electronic structure using density differentiation. Nature Nanotech. 1, 60-65 (2006).

19. Cao, Q. et al. Medium-scale carbon nanotube thin-film integrated circuits on flexible plastic substrates. Nature 454, 495-500 (2008).

20. Sun, D. et al. Flexible high-performance carbon nanotube integrated circuits. Nature Nanotech. 6, 156-161 (2011).

21. Cao, Q. \& Rogers, J. A. Ultrathin films of single-walled carbon nanotubes for electronics and sensors: A review of fundamental and applied aspects. Adv. Mater. 21, 29-53 (2009).

22. Zhang, J. et al. Separated carbon nanotube macroelectronics for active matrix organic light-emitting diode displays. Nano Lett. 11, 4852-4858 (2011).

23. Tang, C. W. \& VanSlyke, S. A. Organic electroluminescent diodes. Appl. Phys. Lett. 51, 913-915 (1987).

24. Hussain, M., Choa, Y-H. \& Niihara, K. Conductive rubber materials for pressure sensors. J. Mater. Sci. Lett. 20, 525-527 (2001).

25. Shimojo, M., Namiki, A., Ishikawa, M., Makino, R. \& Mabuchi, K. A tactile sensor sheet using pressure conductive rubber with electrical-wires stitched method. IEEE Sensors J. 4, 589-596 (2004).

26. McAlpine, M. C., Ahmad, H., Wang, D. \& Heath, J. R. Highly ordered nanowire arrays on plastic substrates for ultrasensitive flexible chemical sensors. Nature Mater. 6, 379-384 (2007).

27. Tian, B. Z. Three-dimensional, flexible nanoscale field-effect transistors as localized bioprobes. Science 329, 830-834 (2010)

28. Ko, H. C. et al. A hemispherical electronic eye camera based on compressible silicon optoelectronics. Nature 454, 748-753 (2008).
29. Ramuz, M., Tee, B. C-K., Tok, J. B-H. \& Bao, Z. Transparent, optical, pressure sensitive artificial skin for large-area stretchable electronics. Adv. Mater. 24, 3223-3227 (2012).

30. Yamada, T. et al. A stretchable carbon nanotube strain sensor for human-motion detection. Nature Nanotech. 6, 296-301 (2011).

\section{Acknowledgements}

This work was funded by DARPA/DSO Maximum Mobility and Manipulation. OLED processing was performed as a user project in the Molecular Foundry, supported by the Office of Science, Office of Basic Energy Sciences, of the US Department of Energy under Contract No. DE-AC02-05CH11231. Some of the materials and optical characterization was performed in the Electronic Materials Laboratory at LBNL, which is supported by the Director, Office of Science, Office of Basic Energy Sciences, Materials Sciences and Engineering Division, of the US Department of Energy under Contract No. DE-AC02-05CH11231. A.J. acknowledges support from the World Class University programme at Sunchon National University.

\section{Author contributions}

C.W. and A.J. conceived the idea and designed the experiments. C.W. carried out the device fabrication and electrical characterization. C.W., D.H., Z.Y., J.P., T.C. and B.M. contributed to the OLED fabrication and characterization. K.T. helped with the shadow mask fabrication. C.W., Z.Y. and A.J. contributed to analysing the data. C.W. and A.J. wrote the paper and all authors provided feedback.

\section{Additional information}

Supplementary information is available in the online version of the paper. Reprints and permissions information is available online at www.nature.com/reprints. Correspondence and requests for materials should be addressed to A.J.

\section{Competing financial interests}

The authors declare no competing financial interests. 


\section{User-interactive electronic-skin for instantaneous pressure visualization}

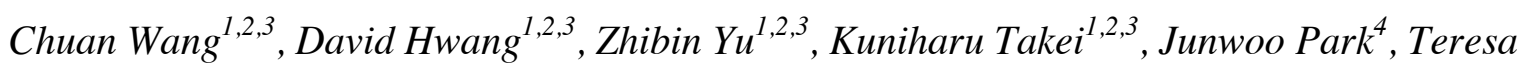

Chen $^{4}$, Biwu $M a^{3,4}$, and Ali Javey ${ }^{1,2,3, *}$

${ }^{1}$ Department of Electrical Engineering and Computer Sciences, University of California, Berkeley, CA 94720

${ }^{2}$ Berkeley Sensor and Actuator Center, University of California, Berkeley, CA 94720

${ }^{3}$ Materials Sciences Division, Lawrence Berkeley National Laboratory, Berkeley, CA 94720

${ }^{4}$ The Molecular Foundry, Lawrence Berkeley National Laboratory, Berkeley, CA 94720

*Correspondence should be addressed to A.J. (ajavey@eecs.berkeley.edu) 


\section{S1. Details about fabrication process for the user-interactive electronic skin}

The detailed fabrication steps are summarized below:

1. Spin coat polyimide (HD MicroSystems, Inc. PI-2525) twice on the silicon handling wafer with a speed of $2000 \mathrm{rpm}$. Cure the polyimide at $300{ }^{\circ} \mathrm{C}$ for 1 hour with $5{ }^{\circ} \mathrm{C}$ per minute ramp rate starting from room temperature. The resulting substrate thickness is $\sim 24 \mu \mathrm{m}$.

2. Photolithography to define the gate electrodes (scan lines). E-beam evaporation of $\mathrm{Ti} / \mathrm{Au}$ $(5 / 35 \mathrm{~nm})$ followed by lift-off in acetone.

3. Deposit $60 \mathrm{~nm}$ of $\mathrm{Al}_{2} \mathrm{O}_{3}$ using atomic layer deposition at $200{ }^{\circ} \mathrm{C}$ and $5 \mathrm{~nm}$ of $\mathrm{SiO}_{\mathrm{x}}$ using ebeam evaporation.

4. Surface functionalization and carbon nanotube network deposition.

a) Immerse the sample into poly-L-lysine $(0.1 \% \mathrm{wt}$ in water from Sigma Aldrich) solution for 5 minutes to functionalize the $\mathrm{SiO}_{\mathrm{x}}$ surface followed by DI water rinse.

b) Next, immerse the amine-functionalized sample into the commercially available 0.01 $\mathrm{mg} / \mathrm{mL} 99 \%$ semiconducting nanotube solution (NanoIntegris Inc.) for 15 minutes followed by DI water and isopropanol rinse, and blow dry with nitrogen.

c) Anneal in vacuum oven at $200{ }^{\circ} \mathrm{C}$ for $1 \mathrm{~h}$ to further clean the surface and remove surfactant residues.

5. Photolithography to define the source/drain electrodes (data lines). E-beam evaporation of $\mathrm{Ti} / \mathrm{Pd}(0.5 / 40 \mathrm{~nm})$ followed by lift-off in acetone.

6. Photolithography to define the active channel region. Oxygen plasma (60 W, 180 mTorr, 15 seconds) is used to etch the unwanted carbon nanotubes outside the channel region.

7. OLED integration. 
a) Photolithography to define the anodes of the OLEDs. DC sputtering of $50 \mathrm{~nm}$ ITO (50 W, $10 \mathrm{sccm}$ Ar) followed by lift-off in acetone.

b) Passivate the entire sample with photoresist. Photolithography to define rectangular openings with a size of $0.91 \times 0.67 \mathrm{~mm}^{2}$ on top of the all ITO electrodes.

c) Bake the sample in air at $250{ }^{\circ} \mathrm{C}$ on a hotplate for 30 minutes to anneal the sputtered ITO and hard bake the photoresist.

d) OLED evaporation through a shadow mask with pixel patterns using a high vacuum ( $2 \times 10^{-6}$ mbar) thermal evaporator in a glovebox.

e) Deposition of $50 \mathrm{~nm}$ of 4-4'-bis[N-(1-naphthyl)-N-phenyl-amino]biphenyl (NPD), $40 \mathrm{~nm}$ of emissive layer, $1 \mathrm{~nm}$ of lithium fluoride (LiF) and $100 \mathrm{~nm}$ of Al consecutively for OLEDs.

8. The fully fabricated sample is delaminated from the silicon handling wafer.

9. Ag ink is used to coat one side of the pressure sensitive rubber (PSR) to work as the ground electrode. The PSR (PCR Technical, JAPAN) is laminated on top of the OLEDs to work as the pressure sensors.

The above steps conclude the fabrication of the user-interactive electronic skin. 

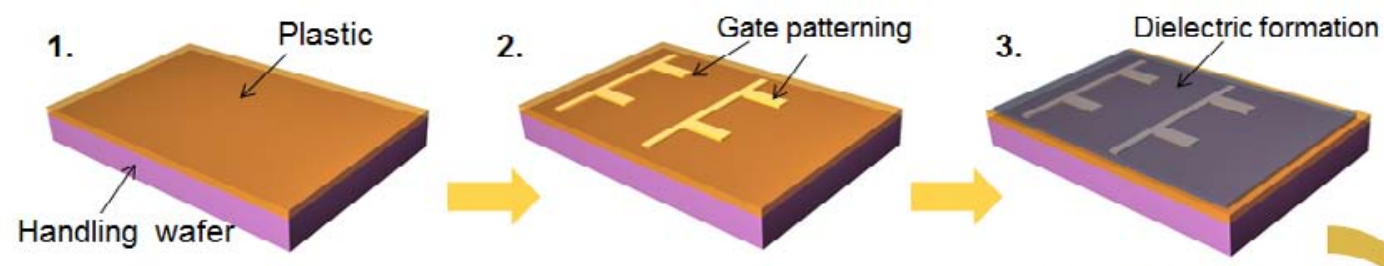

Surface modification
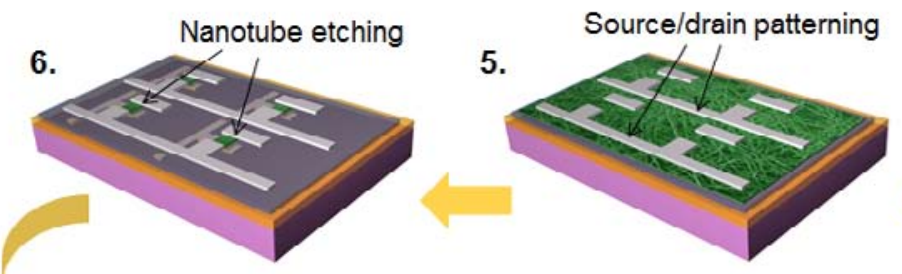

\& nanotube deposition

4.
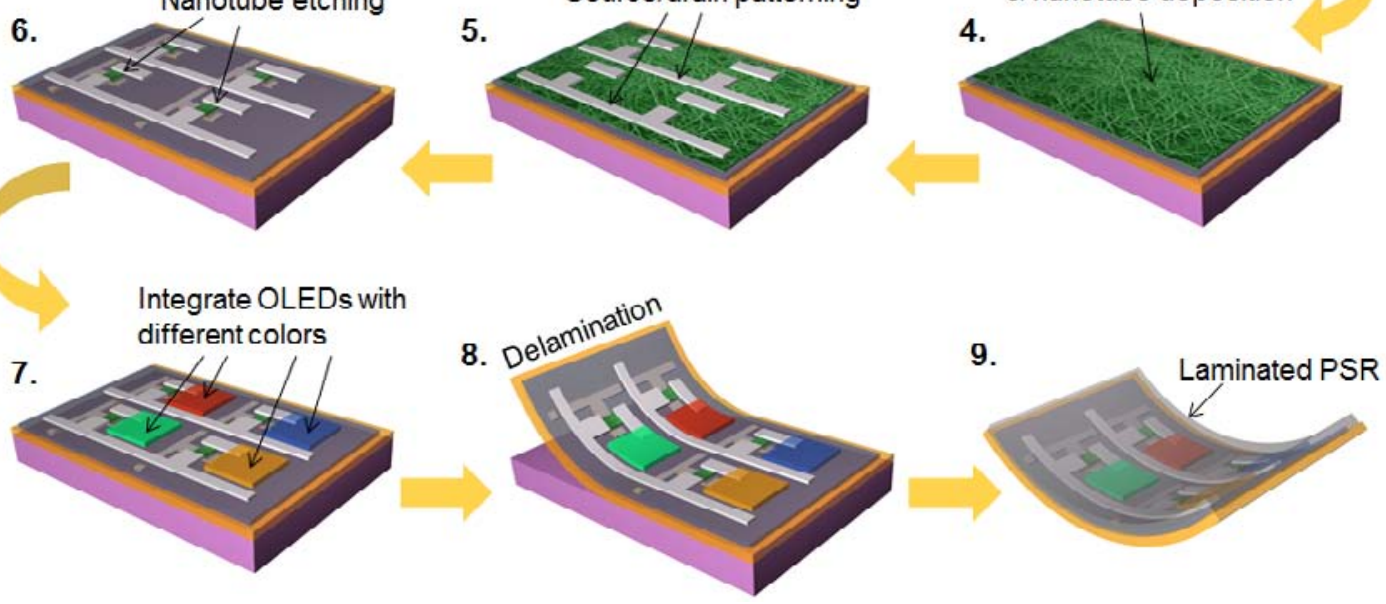

Figure S1. Schematic illustration of the fabrication process for the user-interactive electronic skin. 


\section{S2. Electrical characteristics of the carbon nanotube TFTs}

Representative transfer and output characteristics of nanotube TFTs in the active-matrix backplane are shown in Fig. S2a and b. The histograms for various device parameters are shown in Figures S2c and d.

Given the high transconductance and mobility of nanotube TFTs (Fig. S2d), high current levels can be obtained at relatively low voltages (Fig. S2a-b). The current drives required for obtaining a specific brightness vary depending on the particular OLED colour used in our study as can be seen from Figures $2 \mathrm{c}$ and $2 \mathrm{~d}$. Using the blue OLED as an example, it can be deduced that a current level of $\sim 200 \mu \mathrm{A} / \mathrm{mm}^{2}$ is required to obtain a brightness of $\sim 100 \mathrm{Cd} / \mathrm{m}^{2}$. The average on-current density of the nanotube TFTs is $\sim 3.6 \mathrm{~mA}$ at $V_{\mathrm{DD}}=5 \mathrm{~V}$ for a channel length of $\mathrm{L} \sim 20 \mu \mathrm{m}$ and width of $\mathrm{W} \sim 2000 \mu \mathrm{m}$. The unit-area $(\mathrm{W} \times \mathrm{L})$ normalized on-current for the TFTs is thereby $\sim 90 \mathrm{~mA} / \mathrm{mm}^{2}$, which is $\sim 450 \times$ higher than what is needed to obtain $\sim 100 \mathrm{Cd} / \mathrm{m}^{2}$ from a blue OLED. The high current drive of the TFTs, enables dedicating smaller pixel area to the TFTs as compared to the OLEDs. In this work, we used $W \times L=0.04 \mathrm{~mm}^{2}$ for the TFTs for a pixel size of $\sim 1 \mathrm{~mm}^{2}$ (OLED size $\left.\sim 0.6 \mathrm{~mm}^{2}\right)$.

The average off-state current of TFTs is $550 \mathrm{nA}$ per pixel (Fig. 2a). This off-state current, while low enough for the system demonstration of this work, may be further improved in the future by the use of higher semiconductor-enriched nanotube samples ( $99 \%$ was used in the current work). The total static power consumption of the e-skin matrix caused by the off-state leakage current of the TFTs is estimated to be $\sim 1.4 \mathrm{~mW}$ for a $V_{\mathrm{DD}}$ of $10 \mathrm{~V}$. 

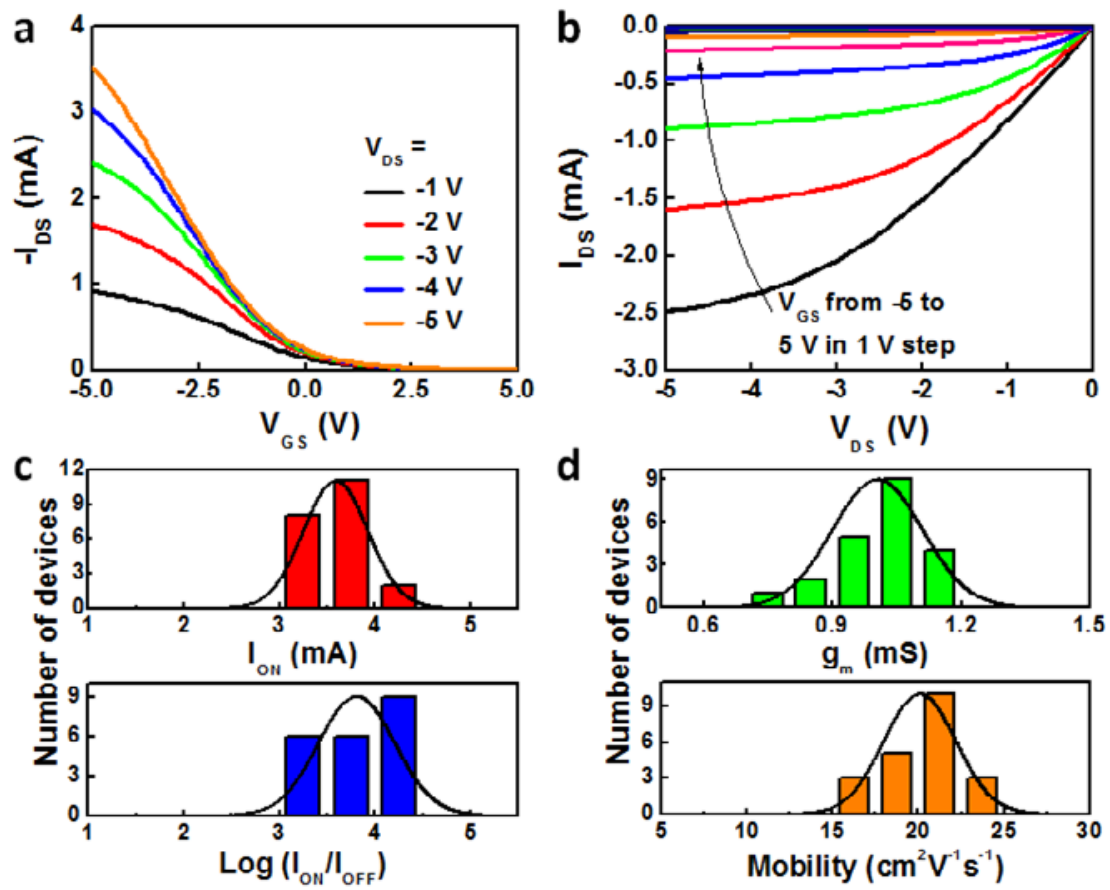

Figure S2 . (a, b) Representative $I_{\mathrm{DS}}-V_{\mathrm{GS}}$ and $I_{\mathrm{DS}}-V_{\mathrm{DS}}$ characteristics of a flexible carbon nanotube TFT $(L=20 \mu \mathrm{m}$, $W=2000 \mu \mathrm{m})$ used in the interactive electronic skin. (c, d) Histograms of the various device performance metrics (on-current, on/off current ratio, transconductance, and field-effect mobility). 


\section{S3. Mechanical bending tests}

Carbon nanotube TFTs and OLEDs can be bent to a curvature radius of $\sim 4 \mathrm{~mm}$ without significant change in the electrical characteristics as depicted in Fig. S3a-b. Figure S3c depicts the proper operation of the e-skin system while bent. It is clear that only pixels where the surface is locally touched are turned ON (i.e., light is locally emitted) with the other pixels remaining OFF.
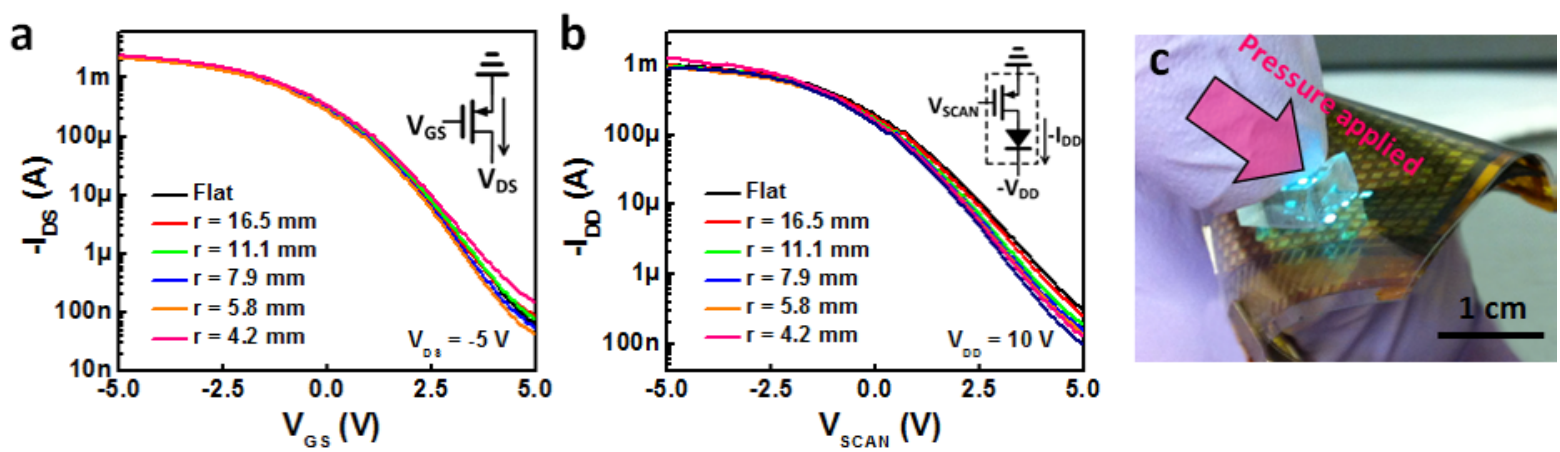

Figure S3. (a) $I_{\mathrm{DS}}-V_{\mathrm{GS}}$ characteristics of a carbon nanotube TFT measured at various curvature radii. (b) $I_{\mathrm{DD}^{-}}$ $V_{\text {SCAN }}$ characteristics of a single pixel circuit (consisting of a TFT and OLED) measured at various curvature radii. (c) Photograph of a fully integrated system showing the device functions properly even under bending conditions. 


\section{S4. OLED structures}

a
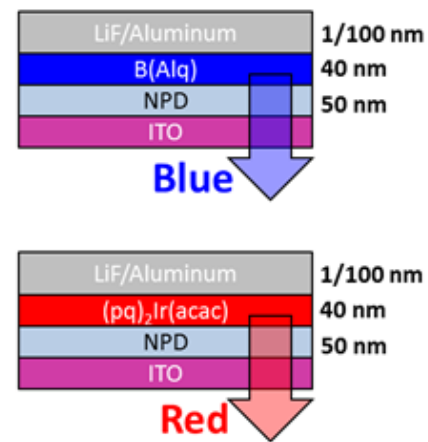

b
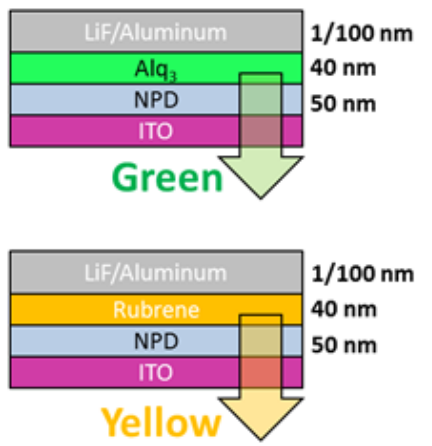
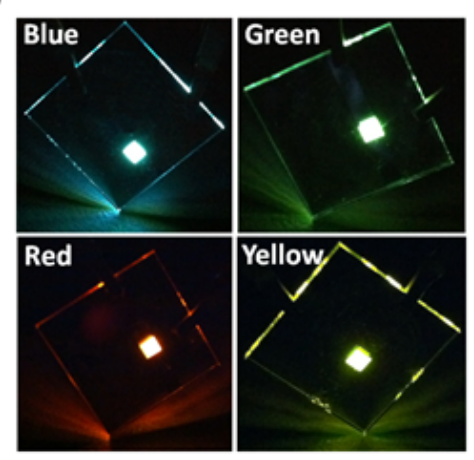

Figure S4. (a) Structures of OLEDs with four different colours used in the interactive electronic skin. (b) Optical photographs of standalone OLEDs being turned ON, corresponding to the structures shown in Figure S4a. 


\section{S5. Pressure response of the PSR and PSR+OLED combination}

In our current work, $\sim 8.5 \mathrm{kPa}$ of applied pressure is necessary to produce visible output signal (i.e., $>1 \mathrm{Cd} / \mathrm{m}^{2}$ ) from the OLEDs as depicted in Fig. 4. However, both the current and brightness of the OLEDs respond to pressure as small as $1 \mathrm{kPa}$ as can be seen in the log-scale plots (Fig. S5b-c). This sensitivity limit is close to that of the PSR (Fig. S5a).

In the future, by improving the OLEDs, the brightness of the pixels at lower applied pressures can be further enhanced if desired. Specifically, here, a basic bilayer OLED structure was used for demonstration purposes, which has a luminescence efficiency of $\sim 1 \%$ (Fig. S5d). In the future, one can adopt more sophisticated OLED structures with better efficiencies (e.g. up to $\sim 20 \%$ or higher as shown previously in the literature $)^{31}$. This would proportionally reduce the required current drive of the OLEDs for the same output light intensity; thereby, increasing the brightness of the pixels for the low pressure range. 

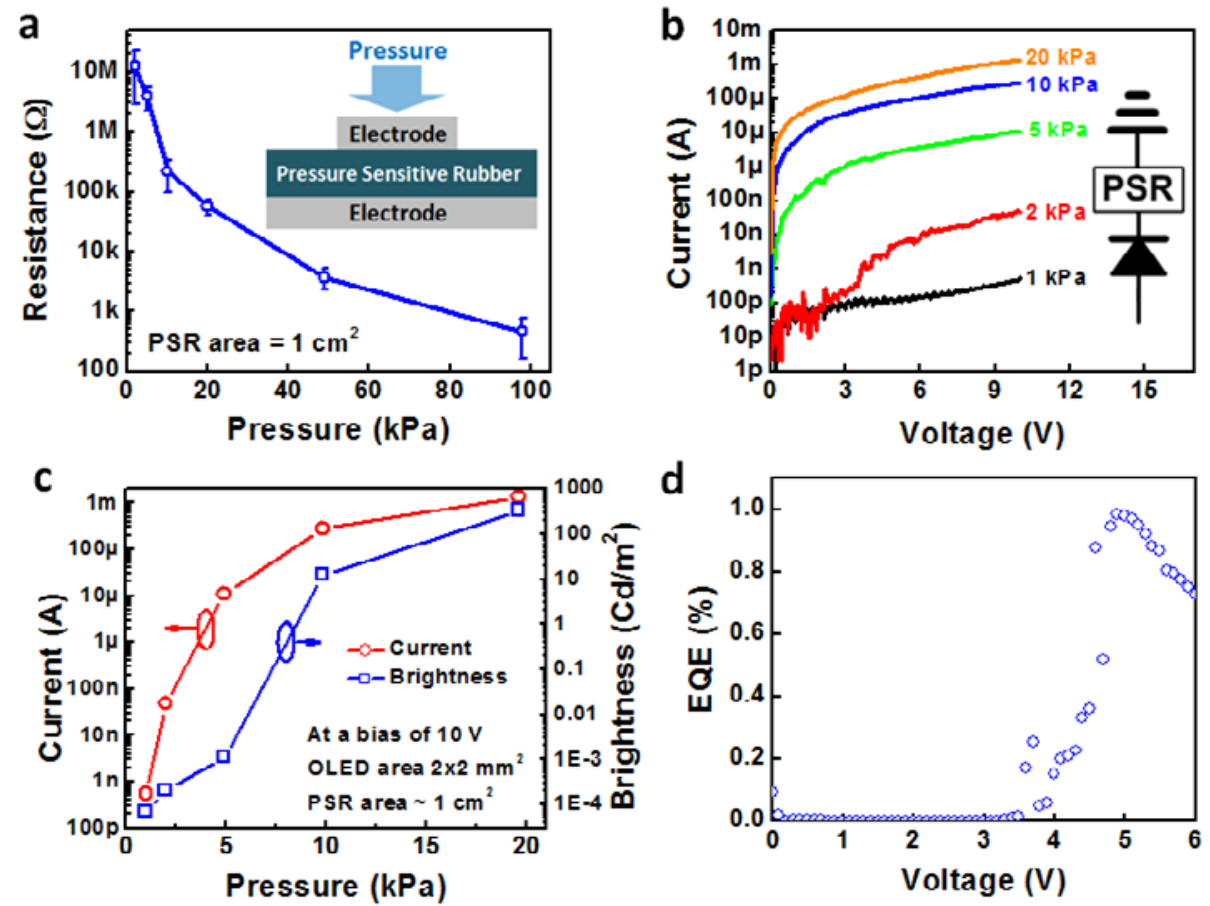

Figure S5. (a) Resistance as a function of applied pressure for the PSR. Inset: schematic showing the measurement setup. The top electrode has an area of $1 \mathrm{~cm}^{2}$. (b) Log-scale $I-V$ characteristics of a blue OLED and PSR combination under various applied pressures. (c) Log-scale current (red trace) and brightness (blue trace) of an OLED/PSR combination circuit as a function of applied pressure. Note that (b) and (c) are the log-scale plots of the data shown in Figs. 4b-c of the main text. (d) External quantum efficiency of a representative blue OLED used in this work. 


\section{S6. Electrical readout of the user-interactive e-skin}

In addition to the optical readout, the electrical readout is also possible with the userinteractive e-skin. The user-interactive e-skin system is made to work in two different ways. For the optical readout, all the scan and data lines are connected to -5 and $10 \mathrm{~V}$ (Fig. $5 \mathrm{~b}$ in the main paper), respectively, to turn on the carbon nanotube TFTs in all the pixels simultaneously so that the output pattern can been seen even without fast line-by-line scan. Although the sample now works in a similar way as a simpler OLED+PSR system, the benefit of the carbon nanotube TFTs is that they could still enable us to selectively disable part of the sensor array if necessary. On the other hand, for the electrical measurements, the current flowing through each pixel is measured individually by applying $-5 \mathrm{~V}$ on the scan line (gate) and $10 \mathrm{~V}$ as the $V_{\mathrm{DD}}$. After scanning all 256 pixels, the current data is plotted as a 2-dimensional contour plot shown in Fig. S6a. The electrical readout data correlates well to the optical output results (Fig. S6c).

The readout of both electrical and optical data simultaneously could also be possible but would require additional work in the future. The realization would require fast refresh rate lineby-line scan so that all the active OLEDs can be visible to the human eye simultaneously. Just like the operation of a regular display, the user-interactive e-skin array could be scanned by selecting only one row $\left(V_{\mathrm{G}}=-5 \mathrm{~V}\right)$ at a time and disabling all the others $\left(V_{\mathrm{G}}=5 \mathrm{~V}\right)$. All the columns would be connected to $V_{\mathrm{DD}}=10 \mathrm{~V}$ with separate channels to monitor the current in each column. This should enable both electrical and optical output simultaneously as long as a reasonably fast refresh rate (e.g. $>60 \mathrm{~Hz}$ ) is used. Although the response time of the individual pixels in our user-interactive e-skin is fast enough ( $1 \mathrm{~ms}$; Fig. S7) to meet the above requirement, one has to make sure that the entire system can also be operated at high scan rates 
considering the RC delay introduced in the interconnections. This is currently not the case. In the future, more optimized system design with reduced parasitic capacitances (for instance by using thicker insulating layers between the crossing metal lines) can lead to higher response rates.
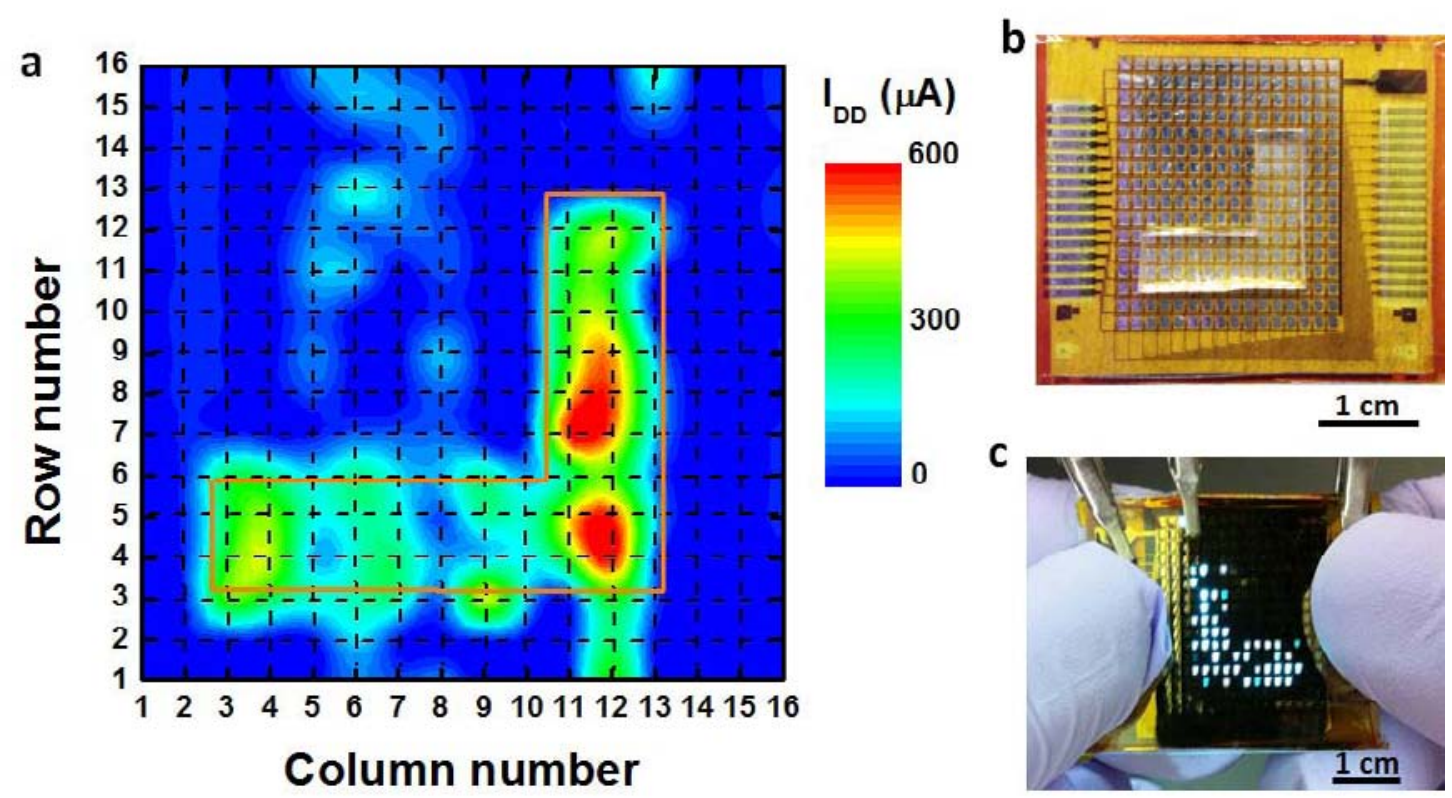

Figure S6. (a) The two-dimensional electrical current profile obtained from experimental mapping of all the pixels individually. (b) L-shaped PDMS is used to apply pressure onto the user-interactive e-skin array for the electrical readout experiment. $200 \mathrm{~g}$ of weight is placed onto the PDMS with a size of around $0.5 \mathrm{~cm}^{2}$ and the corresponding pressure is $39.2 \mathrm{kPa}$. (c) The corresponding optical output from the same system. 


\section{S7. Time response of the single pixel circuit}

The cutoff frequency $\left(f_{\mathrm{t}}\right)$ of the carbon nanotube TFTs used in this work has been characterized in our previous publication ${ }^{15}$. The $f_{\mathrm{t}}$ of the nanotube transistors was measured to be $170 \mathrm{MHz}$ for transistors with a channel length of $4 \mu \mathrm{m}$. For long-channel field-effect transistor, the $f_{\mathrm{t}}$ can be considered inversely proportional to channel length squared $\left(L^{2}\right)$. Therefore, for the nanotube transistors used in the active-matrix backplane in this paper, the cutoff frequency is estimated to be around $6.8 \mathrm{MHz}$ for a channel length of $20 \mu \mathrm{m}$. This speed is fast enough for most active-matrix backplane applications.

In order to characterize the response time of a single pixel circuit consisting of a TFT and an OLED, one pixel is connected to an external resistor (Fig. S7a). The resistance value of $1 \mathrm{M} \Omega$ is chosen so that it is in between the on- and off-state resistance of the TFT/OLED combination. A $V_{\mathrm{DD}}$ of $10 \mathrm{~V}$ is used just like the user-interactive e-skin system. Function generator is used to supply square wave inputs (-5 to $5 \mathrm{~V}$ rail-to-rail) to the gate of the carbon nanotube TFT, and an oscilloscope is used to obtain the waveform from the output node. From the results, the response time of the pixels is estimated to be around $1 \mathrm{~ms}$.

We note that the operating speed of the single pixel circuit is slower than the intrinsic performance of nanotube TFTs $(\sim 7 \mathrm{MHz})$, which is extracted after de-embedding all the parasitic capacitances. In the real-world operation of the user-interactive e-skin, the parasitic capacitance from the metal interconnections and large size OLEDs used in the system $\left(0.91 \times 0.67 \mathrm{~mm}^{2}\right)$ causes significant RC delay and reduces the maximum operating speed of the system to $\sim 1 \mathrm{kHz}$. However, this speed would still be fast enough for most practical sensing and mapping applications. 
a

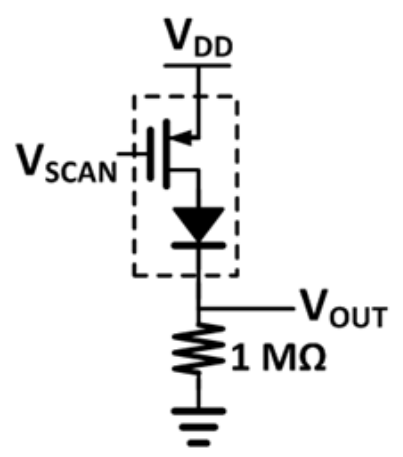

C

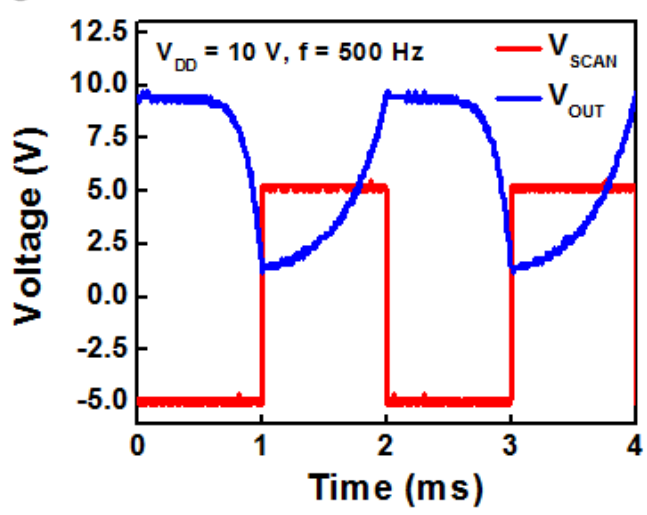

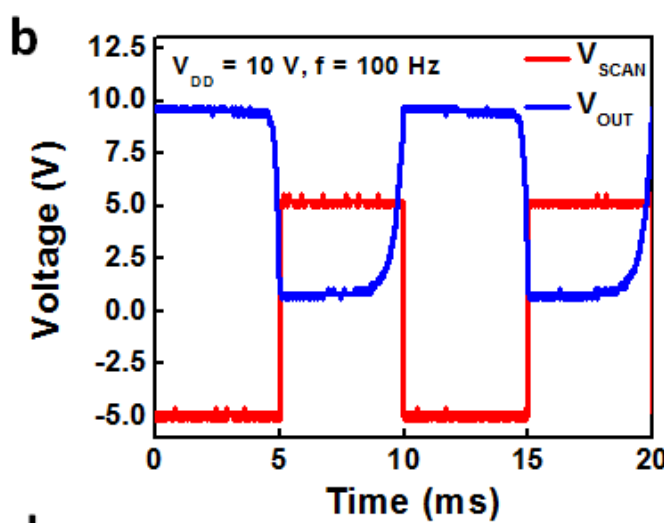

d

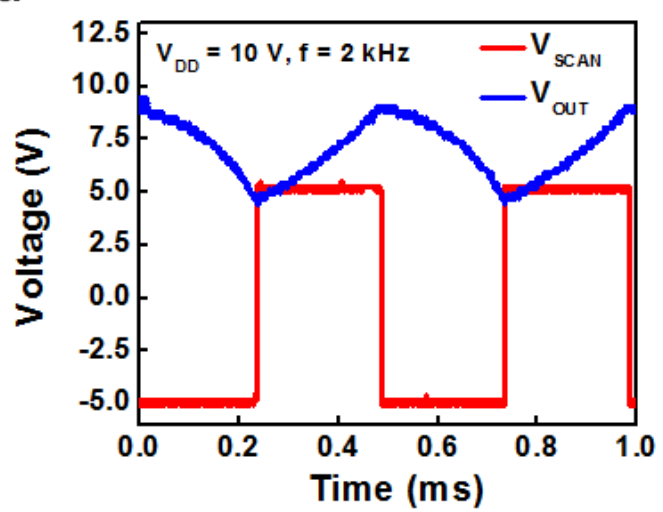

Figure S7. (a) Circuit schematic used to measure the time response of the single pixel circuit consisting of a carbon nanotube TFT and an OLED. (b) Input/output waveforms of the circuit measured at $100 \mathrm{~Hz}$. (c) Input/output waveforms of the circuit measured at $500 \mathrm{~Hz}$. (d) Input/output waveforms of the circuit measured at $2 \mathrm{kHz}$. 


\section{S8. Time response of the user-interactive electronic skin}

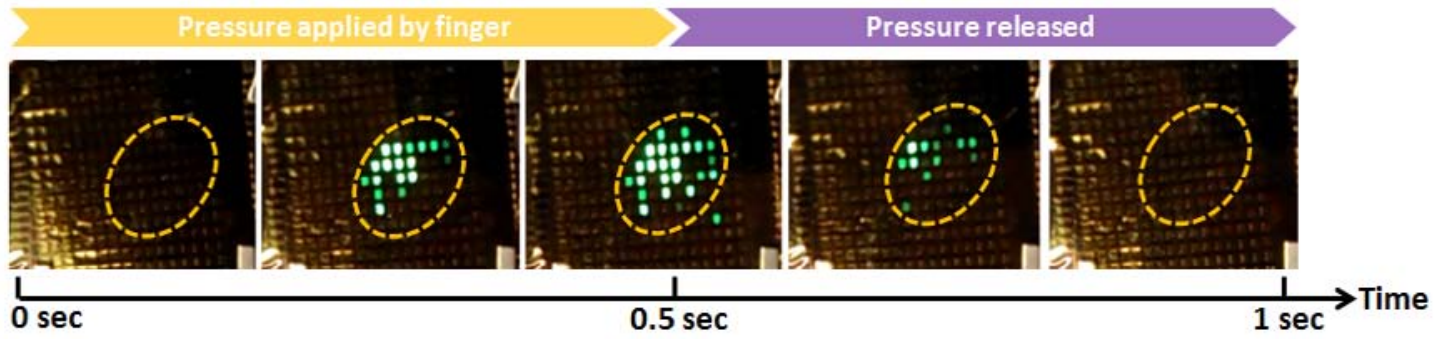

Figure S8. A series of photographs showing selected pixels turned on when the sample is pressed by a finger from the back side. The sample can be operated at speeds well above $1 \mathrm{~Hz}$. 


\section{S9. OLED encapsulation using parylene}

We have performed preliminary experiments to demonstrate the feasibility of encapsulating the OLEDs without compromising the bendability of the system. As an example, we have encapsulated green OLEDs on a polyimide substrate using parylene ${ }^{32}$ (parylene-C with a thickness of $1.5 \mu \mathrm{m})$. First of all, parylene does not compromise the mechanical flexibility of the OLEDs as shown in Fig. S9a, which is expected given that parylene is a polymer. In addition, the parylene encapsulated OLEDs exhibit significantly improved lifetime as compared to the OLEDs without encapsulation (Fig. S9b and c). We do note that parylene encapsulation is far from being ideal and the lifetime of the encapsulated OLED is still limited to a few weeks. Therefore, developing a robust encapsulation scheme for flexible OLEDs and the user-interactive e-skin system, although well-beyond the main scope of this paper, remain to be important and will still require significant research efforts in the future.
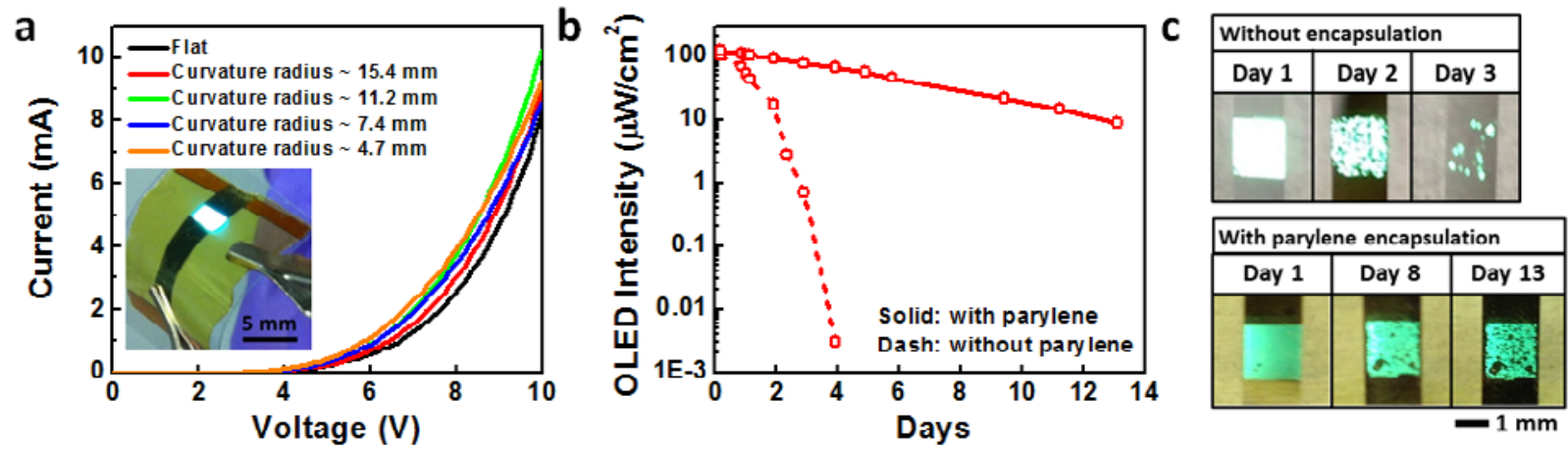

Figure S9. (a) $I-V$ characteristics of a parylene-encapsulated green OLED measured under various bending radii showing that parylene does not compromise the mechanical flexibility of the device. Inset: optical photograph of the flexible OLED. (b) Measured output light intensity as a function exposure time to air for the OLEDs with and without parylene-C encapsulation. Both OLEDs were kept in air. The voltage $(10 \mathrm{~V})$ was applied only during the measurements. (c) Optical photographs showing the visual appearance of the OLEDs in the on-state with (bottom) and without (top) parylene encapsulation as a function of exposure time to air. 


\section{Supplementary References}

31. Sasabe, H. \& Kido, J. Multifunctional materials in high-performance OLEDs: challenges for solid-state lighting. Chem. Mater. 23, 621-630 (2011).

32. Kho, S., Cho, D. \& Jung, D. Passivation of organic light-emitting diodes by the plasma polymerized para-xylene thin film. Jpn.J. Appl. Phys. 41, 1336-1338 (2002). 